PRAXIS

educativa

Universidad Nacional de La Pampa

Facultad de Ciencias Humanas

Instituto de Ciencias de la Educación

para la investigación interdisciplinaria

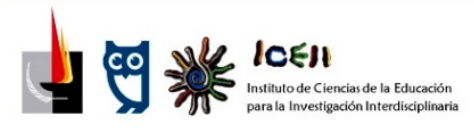

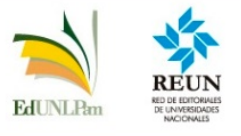

ISSN 2313-934X

SANTA ROSA, LA PAMPA, ARGENTINA

Correo electrónico: iceii@humanas.unlpam.edu.ar

Disponible en https://cerac.unlpam.edu.ar/index.php/praxis

Evaluación de la percepción de redes sociales desde el Modelo de Aceptación Tecnológica en estudiantes en línea de psicología mexicanos de José Manuel Meza Cano; Edith González Santiago. Praxis educativa, Vol. 26, No 1 enero - abril 2022. E - ISSN 2313-934X. pp. 1-16. https://dx.doi.org/10.19137/praxiseducativa-2022-260106

\title{
Evaluación de la percepción de redes sociales desde el Modelo de Aceptación Tecnológica en estudiantes en línea de psicología mexicanos ${ }^{\mathrm{i}}$
}

Evaluation of perception of social network from the technological acceptance model in Mexican online Psychology students

Avaliação da percepção das redes sociais desde o Modelo de Aceitação Tecnológica em estudantes online de psicologia mexicanos

\section{José Manuel Meza Cano}

Universidad Nacional Autónoma de México, México

manuel.meza@iztacala.unam.mx

ORCID 0000-0002-9504-7906

\section{Edith González Santiago}

Universidad Nacional Autónoma de México, México

edith.gonzalez@iztacala.unam.mx

ORCID 0000-0002-7876-6079

Recibido: 2021-02-19 | Revisado: 2021-07-06 | Aceptado: 2021-08-17 


\section{Resumen}

El objetivo fue evaluar y describir la percepción sobre redes sociales desde el Modelo de Aceptación Tecnológica para describir la actitud, la utilidad percibida, la facilidad de uso, la confianza y el riesgo percibido de las redes sociales empleadas por estudiantes en línea de psicología. El estudio fue de tipo no experimental, transeccional, con alcance correlacional. La muestra fue no probabilística de 74 estudiantes de diferentes regiones de México con una media de edad de 35 años; de ellos, 56 fueron mujeres. Se utilizó el Instrumento de Aceptación Tecnológica (TAM) adaptado a redes sociales virtuales. Los resultados muestran que Facebook es la red social más empleada. Las escalas de Riesgo y Confianza mostraron medias moderadas; el resto mostró medias altas. Se encontró que, a mayor edad, disminuye la percepción de Facilidad de uso. Las mujeres mostraron una mayor percepción de Riesgo y los usuarios de Facebook mostraron una menor percepción de Utilidad.

Palabras clave: Redes sociales; Internet; Percepción; Actitud; Tecnología de la comunicación.

\section{Abstract}

The objective was to evaluate and describe the perception of social networks from the Technological Acceptance Model to describe the attitude, perceived usefulness, ease of use, trust, and perceived risk of social networks used by online psychology students. The study was non-experimental, transactional, with correlational scope. It was a non-probabilistic sample of 74 students from different regions of Mexico with an average age of 35 years, of which 56 were women. The Technological Acceptance Instrument (TAM) adapted to virtual social networks was used. The results show that Facebook is the most widely used social network. The Risk and Trust scales showed moderate means, the rest showed high means. It was found that at an older age the perception of Ease of use decreases. Women showed a higher perception of Risk and Facebook users showed a lower perception of Utility.

Keywords: Social media; Internet; Perception; Attitude; Communication technology.

\section{Resumo}

O objetivo foi avaliar a percepção dos estudantes de psicologia na modalidade online sobre as redes sociais utilizando o Modelo de Aceitação Tecnológica. 0 propósito foi descrever sua atitude sobre e sua confiança nas redes sociais, além da utilidade e risco percebidos e facilidade de uso das redes empregadas por eles. Foi uma pesquisa não-experimental, transversal e correlacional. Utilizou-se amostras não probabilística de 74 estudantes de diferentes regiões do México, sendo 56 mulheres. A idade média foi de 35 anos. Aplicou-se o Instrumento de Aceitação Tecnológica (TAM) adaptado às redes sociais virtuais. Os resultados demonstram que Facebook é a rede mais utilizada. As escalas de Risco e Confiança mostraram médias moderadas, o resto das escalas teve médias altas. Encontrou-se ainda que quanto maior a idade, menor a percepção de Facilidade de uso. As mulheres demonstraram maior percepção de Risco e, os usuários do Facebook, menor percepção de Utilidade.

Palavras chave: Redes sociais; Internet; Percepção; Atitude; Tecnologia de comunicação. 


\section{Introducción}

Las redes sociales son uno de los medios de comunicación más importantes hoy en día. En ellas, las personas envían información, comparten ideas e inquietudes y se comunican con amistades y familiares. Desde el punto de vista educativo, pueden ser un medio a través del cual estudiantes y profesores pueden crear un entorno de aprendizaje colaborativo, dada su creciente importancia, aceptación y uso. A pesar de esto, cabe preguntarse: ¿qué redes sociales? Si bien redes como Facebook, Twitter o Instagram son las más empleadas, no siempre son las más seguras o las que se perciben con un menor riesgo desde el punto de vista de los estudiantes. Por tanto, el presente trabajo pretende indagar en la percepción que tienen los estudiantes sobre las redes sociales que emplean, partiendo del Modelo de Aceptación Tecnológica (TAM). Se inicia el trabajo exponiendo las redes sociales más empleadas por los estudiantes, para luego describir el modelo antes mencionado y algunos trabajos relevantes que han empleado este modelo aplicado en redes sociales. Posteriormente, se describe la recogida de información y el análisis de resultados cuantitativos tanto descriptivos como inferenciales. Para concluir, se discuten los hallazgos más relevantes, en donde se encontró que las variables como edad, género y uso de Facebook inciden en la percepción de facilidad de uso, percepción del riesgo y percepción de utilidad de las redes sociales.

\section{Redes sociales más empleadas por los estudiantes}

Según un estudio sobre los hábitos de los usuarios de Internet de la Asociación Internet MX (2019), el 82\% de los encuestados afirmaron usar Internet para conectarse a las redes sociales. Esta actividad es la más mencionada por los participantes, quienes refieren hacerlo varias veces al día. Por su parte, el Instituto Federal de Telecomunicaciones (2019) menciona que existe un 51.2\% de probabilidades de que en México una persona de seis años o más use redes sociales, siendo la población masculina la que tiene una mayor probabilidad de uso con un $51.5 \%$. En cuanto al nivel educativo de los usuarios de redes sociales, tenemos que, en nivel posgrado, hay una probabilidad de uso de un $85.3 \%$, 34.1 puntos porcentuales arriba del promedio nacional que es de $51.2 \%$, mientras que los estudiantes de licenciatura tienen una probabilidad de uso del $83.4 \%$. Según esta encuesta, los estudiantes son quienes tienen mayor probabilidad de usar redes sociales, con $82.7 \%$ de acuerdo a la ocupación de usuarios. En cuanto a las edades de los usuarios, los de 18 a 24 años son quienes tienen mayor probabilidad de uso de las redes sociales, siendo el $84.7 \%$ de los encuestados. Por su parte, Kemp (2020) muestra, en su encuesta sobre las plataformas, que las más usadas en México son: YouTube, 96\% de los usuarios; Facebook, con 94\%; WhatsApp, 89\%; FB Messenger, 78\%; Instagram, 71\%; Twitter, 61\%; Pinterest, 46\%; Linkedln, 36\%; Snapchat, 35\%; Skype, 32\%; Tumblr, 22\%; Twitch, 22\%; WeChat, 21\%; TikTok, 20\%; Reddit, 17\%; y Line, 16\%.

Hablando del contexto global, una encuesta reportada por Kemp (2020) muestra que Facebook es la plataforma más usada alrededor del mundo con dos mil seiscientos tres millones de usuarios. En segundo lugar, se encuentra YouTube con do mil millones de usuarios activos y, 
José Manuel Meza Cano; Edith González Santiago

en tercer lugar, se encuentra Facebook Messenger, con mil trescientos millones de usuarios activos. En dicha encuesta, se encuentran otras plataformas para socializar que se posicionan en referencia al lugar geográfico de manera global. A esta lista le siguen en orden de número de usuarios WeChat, Instagram, TikTok y Twitter.

Esta misma encuesta menciona que México, dadas sus características sociodemográficas y geográficas, es un referente para medir el avance y crecimiento en el uso de la tecnología y su consumo. Actualmente, existen 128,3 millones de habitantes en México y el 89\% de esa población tiene un teléfono móvil, encontrando que ochenta y nueve millones de mexicanos son usuarios activos de internet, lo que representa el 69\% de la población total. Algo importante de mencionar es que el $89 \%$ de los usuarios de internet han interactuado en redes sociales como prosumidores, es decir que, además de consumir, también crean y comparten contenido, y también participan con intervenciones y en debates. Los usuarios mexicanos invierten tres horas y 25 minutos, aproximadamente, al día en medios sociales, teniendo a veces hasta 10 cuentas en distintas redes de este tipo. Flores et al. (2017) mencionan que, en los últimos años, las redes sociales han permitido a los jóvenes estudiantes universitarios realizar varias actividades sociales a la vez: subir vídeos, compartir fotografías, estados de ánimo, relacionarse con personas distintas, etc., pero, al mismo tiempo, se han ido modificando las formas de comunicación. Las redes sociales pueden significar un entorno favorable para el aprendizaje, ya sea formal o informal; el que sea social podría favorecer "la inteligencia colectiva estableciendo nuevas estructuras de participación, ya que todos aportamos al conocimiento" (Flores et al., 2017, p. 57). A decir de Díaz (2015), las redes sociales tienen tres tareas estructurales: comunicación, cooperación y comunidad, lo que podría explicar el crecimiento en el número de usuarios.

A pesar de que la UNESCO (2014) ha impulsado el uso de la tecnología como una forma de promover la ciudadanía digital entre los jóvenes del mundo, existen factores que, de no atenderse, podrían significar cierta vulnerabilidad en el uso de las redes sociales. Varchetta et al. (2020) mencionan riesgos como deficiencias en el mundo real por la búsqueda continua de experiencias virtuales, uso compulsivo de las redes sociales, presencia del fenómeno llamado FOMO o Miedo a quedarse afuera (Fear of Missing Out), interacciones mediadas por mecanismos de falta de empatía, desconexión moral, deshumanización hacia el interlocutor, ciberbullying o, como lo menciona Guzmán (2019), el robo de información confidencial para distintos usos.

\section{Modelo de Aceptación Tecnológica (TAM)}

Hay varios modelos que observan la aceptación de la tecnología, sin embargo, el más conocido y utilizado es el propuesto por Davis en 1989. Este modelo observa la actitud y predisposición respecto a la intención de uso de una tecnología, esta observación está definida por las variables utilidad percibida y facilidad de uso (Cabero et al., 2018; Vidal, 2018).

El objetivo de este instrumento es ofrecer un marco teórico desde el cual sea posible una explicación de los factores que intervienen en la adopción de las TIC. Este Modelo de aceptación de la tecnología (Technology Acceptance Model, TAM por sus siglas en inglés) es una adaptación 
José Manuel Meza Cano; Edith Conzález Santiago.

de la Teoría de la Acción Razonada (TRA) que, a decir de Vidal (2018), propone que las creencias influyen en la actitud de un sujeto y, por lo tanto, en su intención y comportamiento.

El TAM se basa, fundamentalmente, en tres constructos (Vidal, 2018):

Actitud. Se refiere a la predisposición aprendida para responder favorable o desfavorablemente con respecto a un objeto dado.

Utilidad percibida. Se refiere a la probabilidad subjetiva de una persona de que el uso mejorará su actuación en determinado campo.

Facilidad de uso. Se refiere al grado en que un sujeto percibe que el uso de determinado sistema u objeto está libre de esfuerzo.

Desde esta perspectiva, este modelo muestra que los usuarios de determinado objeto o sistema lo aceptan, o no, en función de las creencias de utilidad y facilidad de uso, lo que impacta en la actitud frente a este y hace posible la predicción de intención de uso.

Aunque es un modelo bastante sólido para explicar la aceptación de la tecnología, numerosas investigaciones lo han explorado en distintos contextos sociodemográficos para observar otros factores implicados en la aceptación de distintas tecnologías y sistemas, se han incluido otras variables como las demográficas, la autoeficacia, la influencia social, la innovación personal, características culturales, el control conductual, factores motivacionales, el carácter lúdico, entre otros, y el TAM ha mostrado solidez y consistencia en la predicción y explicación de los factores involucrados para que un usuario adopte determinados sistemas (Vidal, 2018).

\section{Investigaciones que han empleado el instrumento del Modelo de Aceptación Tecnológica en Redes Sociales}

El TAM ha sido adaptado por Lorenzo et al. (2011) en España, lo modificaron agregando los constructos de confianza y riesgo percibido porque los consideran importantes en los entornos en línea donde la incertidumbre está presente. Estos constructos, afirman los autores, ya habían sido utilizados en el comercio electrónico por Pavlou (2003, citado en Lorenzo et al., 2011), pero no en las Redes Sociales Virtuales (RSV).

El instrumento sobre el TAM ha sido empleado en distintas investigaciones, SánchezFranco et al. (2012) lo usaron para observar la confianza en ambientes en línea como una forma de percibir apoyo comunitario y de la calidad de las relaciones como una forma de establecer compromiso social. Los resultados arrojaron evidencia sobre la promoción de la interdependencia que existe en las redes sociales virtuales entre individuos y su capital social fomentando la participación activa.

En 2015, Sánchez-Franco y Roldán usaron el TAM para observar la relevancia de la familiaridad, la confianza y la reciprocidad en una comunidad virtual como un factor determinante en la construcción de apoyo comunitario percibido. Los resultados mostraron que las normas de reciprocidad impactan en la confianza afectiva y el apoyo comunitario percibido, 
José Manuel Meza Cano; Edith Conzález Santiago

pero no así la familiaridad. Los autores mencionan que estos datos podrían apoyar a proveedores de servicios sociales en línea para motivar el sentido de pertenencia en comunidades virtuales.

En otra investigación llevada a cabo por Luna (2016), se examinaron, mediante el TAM, las variables relacionadas a la intensidad de uso de las redes sociales del consumidor turístico de la generación $Y$. Se encontró que las dos principales razones por las que este tipo de usuario emplea las redes sociales son para buscar información antes y después de vacaciones, así como para crear contenido en la red, también, antes, durante y después de vacaciones. Este estudio concluye, basado en la evidencia y en las investigaciones previas, que el consumidor turístico de la generación $Y$ crea contenido para compartir en redes como una forma de autoexpresión y construcción de identidad mediante las experiencias vacacionales (Consumo Simbólico de la Experiencia Turística).

Otro estudio relevante realizado con el TAM fue el de Peña y Sánchez (2017), quienes siguieron una metodología DBR (Design Based Research) para la implementación de un piloteo con 29 estudiantes universitarios y una herramienta que permite la interacción, evaluación continua e interpretación de datos generados en redes sociales integradas en plataformas de docencia en línea (Tag2Learn). Para la recogida de datos, se utilizaron entrevistas abiertas y un cuestionario tipo Likert de 10 ítems con una escala de cinco puntos que obtuvo datos de la utilidad percibida en el uso de redes sociales en el aula, considerando las dimensiones actitud, valor añadido, intención de uso, riesgo percibido y confianza. Posteriormente, se llevó a cabo un grupo focal basado en el mismo cuestionario donde se discutió la percepción que tenían sobre la contribución a su propio desarrollo en competencias digitales y los elementos transmedia incluidos. Finalmente, el análisis de contenido realizado exploró el uso de las redes sociales en la experiencia formativa. Los resultados mostraron una mejor actitud al uso de la red social Twitter y una intención de uso muy favorable de las redes sociales en los procesos de enseñanza. Mostraron valores que demuestran una tendencia a la percepción alta de riesgo y una baja confianza en referencia al uso de las redes. La valoración a nivel general de la integración de las redes sociales en el aula en el proceso de enseñanza-aprendizaje fue de 4.44 sobre 5 .

De acuerdo con estos antecedentes, modelos basados en el TAM, como el de Lorenzo et al. (2011), en donde se incluye intención de uso, utilidad percibida, facilidad de uso y, además, confianza y riesgo percibido, pueden considerarse como los más completos para indagar acerca de la actitud hacia las redes sociales, dado que mencionan que la actitud es presentada como antecedente fundamental de la intención de uso, tomando en cuenta elementos que pueden influir en ella como la confianza y el riesgo percibido. Considerando estos antecedentes, se pretende indagar sobre estas dimensiones en estudiantes de psicología en línea.

\section{Objetivo}

El objetivo del presente trabajo es describir la actitud, la utilidad percibida, la facilidad de uso, la confianza y el riesgo percibido de las redes sociales empleadas por estudiantes en línea de psicología. Como objetivos específicos, se plantea: evaluar si existen variables sociodemográficas 
que influyen en la actitud, utilidad percibida, facilidad de uso, confianza y el riesgo percibido de las redes sociales y explorar si existen correlaciones entre la actitud, utilidad percibida, facilidad de uso, confianza y riesgo percibido de las redes sociales en esta muestra.

\section{Método}

Se trata de un estudio no experimental, pues no se manipulan las variables; transversal, dado que se recogieron los datos en un único momento; con alcance correlacional, dado que se pretende conocer si algunas características sociodemográficas y del uso de redes sociales impactan en los factores mencionados de las redes sociales empleadas por estudiantes en línea de psicología.

\section{Muestra}

Se trató de una muestra no probabilística, por conveniencia, puesto que los participantes estuvieron inscritos en un curso en línea de psicología extracurricular en los que la respuesta al instrumento formó parte de las actividades a realizar. Participaron 74 estudiantes, con edades de entre 21 y 57 años, una media de 35.12 años, siendo 56 mujeres. De ellos, 51 participantes (68.9\%) pertenecieron a la Zona Metropolitana del Valle de México, que incluye Ciudad de México y Estado de México, mientras que 23 participantes (31.1\%) pertenecieron a otros estados del país como Oaxaca, Chihuahua, Hidalgo, Puebla, Tlaxcala, entre otros. Los estudiantes pertenecían a distintas áreas de psicología, 34 al área de psicología clínica, 15 a psicología educativa, 14 al tronco común de conocimientos básicos en psicología, nueve a psicología de la salud y dos estudiantes pertenecientes al área de Necesidades Educativas Especiales. Los participantes contestaron al instrumento en su totalidad, no hubo datos ausentes.

\section{Instrumento}

Se empleó el Instrumento de Aceptación Tecnológica (TAM) original de Davis (1989), modificado y adaptado al español por Lorenzo et al. (2011) para ser aplicado a redes sociales virtuales, integrando los constructos de confianza y riesgo percibido. Se añadió un consentimiento informado para aclarar que todos los datos se emplean con fines de investigación, se agregó un reactivo para indagar en la red social que emplean los estudiantes con mayor frecuencia y uno para indagar en el dispositivo empleado con mayor frecuencia. Consta de 52 reactivos en siete escalas (ver Tabla 1): 


\section{Tabla 1}

\begin{tabular}{lll}
\hline Estructura del TAM modificado (Lorenzo et al., 2011) & Alfa \\
\cline { 2 - 3 } \# de reactivos & Escala & 0.63 \\
4 & Uso de las redes sociales & 0.87 \\
11 & Utilidad percibida de las redes sociales & 0.88 \\
5 & Facilidad de uso de las redes sociales & 0.94 \\
4 & Actitud hacia las redes sociales & 0.92 \\
13 & Intención de uso de las redes sociales & 0.90 \\
13 & Riesgo percibido de las redes sociales & 0.93 \\
\hline
\end{tabular}

\section{Procedimiento}

El instrumento fue implementado a través de un formulario de Google. Se aplicó en el contexto de un curso en línea de psicología en el cual se solicitó como parte de las actividades, empleando para ello un enlace en la plataforma Moodle que los llevó al instrumento. Dicho instrumento fue contestado por los participantes entre el 20 de marzo y el 8 de abril del 2020. Posteriormente, se descargaron los datos en una hoja de cálculo y se importaron en SPSS V.24 para su análisis estadístico.

\section{Resultados}

A continuación, se muestran los principales resultados iniciando por las preferencias de uso de las redes sociales de los estudiantes para, luego, dar paso a los resultados correlacionales. De los 74 estudiantes, 53 de ellos (71.6\%) reportó emplear Facebook como red principal, seguidos de 13 estudiantes (17.5\%) que prefirieron WhatsApp, cuatro (5.4\%) emplean principalmente Instagram y Messenger; Linkedin, Hotmail y Waze tuvieron únicamente un usuario cada uno (1.3\%).

Como principal herramienta de acceso a la red social, se tuvo que siete participantes (9.4\%) reportaron emplear computadora, mientras que 67 participantes (90.5\%) mencionaron emplear principalmente el teléfono móvil para acceder a las redes sociales.

Se les solicitó a los estudiantes que contestaran el resto del instrumento teniendo en cuenta la red social que emplean con más frecuencia.

Respecto a la escala de Uso de las redes sociales del instrumento, esta no arrojó información sobre consistencia interna a través del Alfa de Cronbach, pero se obtuvieron los siguientes resultados. Al hablar de la frecuencia de uso, se obtuvo que 55 estudiantes (74.3\%) la 
emplean varias veces al día, seguido de 11 (14.9\%) que mencionaron emplearla al menos una vez al día; mientras que cuatro estudiantes (5.4\%) mencionaron usarla al menos una vez a la semana, solo dos (2.7\%) la emplearon dos o tres veces a la semana y un (1.4\%) estudiante mencionó emplearla menos de una vez a la semana.

Al preguntarles sobre la media de horas que emplean la red social cada semana, se obtuvo que 17 estudiantes (23\%) mencionaron emplearlas entre una y cinco horas; 15 (20\%) afirmaron que entre 5 y 10 horas; 12 (16.2\%) las emplearon entre 10 y 15 horas; 11 estudiantes (14.9\%) entre 15 y 20 horas; 9 (12.2\%) entre 20 y 25 horas; 8 (10.8\%) más de 25 horas y solo dos estudiantes (2.7\%) las emplearon menos de una hora a la semana.

Respecto a las demás escalas del instrumento, se obtuvieron los índices de consistencia interna por cada una, junto con sus medias y desviaciones estándar (Tabla 2).

\section{Tabla 2}

Alfa, media y desviación estándar de cada escala del instrumento

\begin{tabular}{llll}
\hline Escala & Alfa & Media & Desv. est. \\
\hline Utilidad percibida de las redes sociales & 0.67 & 3.76 & 0.71 \\
Facilidad de uso de las redes sociales & 0.90 & 4.21 & 0.64 \\
Actitud hacia las redes sociales & 0.85 & 3.93 & 0.72 \\
Intención de uso de las redes sociales & 0.82 & 4.15 & 0.77 \\
Riesgo percibido de las redes sociales & 0.83 & 2.79 & 0.68 \\
Confianza hacia las redes sociales & 0.87 & 2.67 & 0.65 \\
\hline
\end{tabular}

Como puede notarse, la escala con el alfa menor fue Utilidad percibida, con 0.67, siendo considerada aún como un índice de confiabilidad suficiente para ser tomada en cuenta, mientras que el resto de las escalas tuvieron índices por encima del 0.80 , por lo que se consideran fiables. Con respecto a las medias por escalas, fueron las escalas de Confianza $M=2.67$ y Riesgo percibido $M=2.79$ aquellas que presentaron medias bajas en una escala Likert de 1 a 5 . En tanto que las escalas con las medias más altas fueron Facilidad de uso $M=4.21$ e Intención de uso con M=4.15.

Se realizó la prueba de Kolmogorov-Smirnov para una muestra y así determinar si los datos son normales. Para ello, se empleó de igual forma la media de cada escala con la totalidad de la muestra. Los datos encontrados fueron los siguientes (Tabla 3): 
Tabla 3

José Manuel Meza Cano; Edith González Santiago

Escalas y coeficientes obtenidos en la prueba de normalidad Kolmogorov-Smirnov

\begin{tabular}{lllllll}
\hline Escala & $\begin{array}{l}\text { Utilidad } \\
\text { Percibida }\end{array}$ & $\begin{array}{l}\text { Facilidad de } \\
\text { Uso }\end{array}$ & Actitud & $\begin{array}{l}\text { Intención de } \\
\text { uso }\end{array}$ & $\begin{array}{l}\text { Riesgo } \\
\text { Percibido }\end{array}$ & Confianza \\
\hline $\begin{array}{l}\text { Estadístico } \\
\text { de prueba }\end{array}$ & 0.15 & 0.14 & 0.12 & 0.16 & 0.06 & 0.06 \\
$\begin{array}{l}\text { Sig asintótica } \\
\text { (bilateral) }\end{array}$ & 0.04 & 0.00 & 0.01 & 0.00 & $0.20^{*}$ & $0.20^{*}$ \\
\hline
\end{tabular}

*Los datos son normales

Según las pruebas de normalidad, cuando los niveles de significancia son $\mathrm{p}<0.05$, se puede decir que existen datos normales, sin embargo, únicamente las escalas Riesgo percibido y Confianza cumplieron con este requisito, por lo que, al encontrar que la muestra no presentó datos normales en la mayoría de las escalas del instrumento, se tomó la decisión de continuar empleando estadística no paramétrica.

Siguiendo esta ruta, se decidió indagar en las variables sociodemográficas y su relación con las escalas del instrumento. Se encontró que no hay diferencias estadísticamente significativas al diferenciar la muestra por zona de residencia: zona metropolitana y resto del país. Para explorar las diferencias de medias entre las edades, se crearon tres rangos de edad a través de la división de la muestra, lo cual permitió realizar un análisis a través de la prueba KruskalWallis para muestras independientes, en donde únicamente la escala de Facilidad de uso mostró datos estadísticamente significativos (Tabla 4).

\section{Tabla 4}

Comparación de medias entre rangos de edad de la muestra para la escala Facilidad de uso, significativos a $p<0.05$

\begin{tabular}{llll}
\hline Rango de edad & $\mathrm{N}$ & Media & Desv. est. \\
\hline $21-30$ & 28 & 4.42 & .47 \\
$31-40$ & 23 & 4.26 & .61 \\
$>41$ & 23 & 3.90 & .74 \\
\hline
\end{tabular}

Como puede notarse, conforme aumenta la edad, disminuyó la percepción sobre la Facilidad de uso, siendo las personas con más de 41 años las que presentaron medias menores $M=3.90$, mientras que las personas entre 21 y 30 años presentaron una media de $M=4.42$, existiendo una diferencia significativa a $p<0.05$. 
Continuando con estadística no paramétrica, se obtuvieron diferencias estadísticamente significativas en la escala de Riesgo percibido y de Utilidad percibida, como se muestra en la Tabla 5 a través de la prueba $U$ de Mann-Whitney.

\section{Tabla 5}

Prueba U de Mann-Whitney para muestras independientes significativas con $p<0.05$ en las escalas Riesgo percibido y Utilidad percibida

\begin{tabular}{llllll}
\hline Escala & Variable de agrupación & Variable & Media & N & Desv. est. \\
\hline Riesgo percibido & Género & Mujeres & 2.88 & 56 & 0.69 \\
& Hombres & 2.52 & 18 & 0.62 \\
Utilidad percibida & $\begin{array}{l}\text { Usuarios de } \\
\text { Facebook }\end{array}$ & Sí & 3.66 & 54 & 0.70 \\
& No & 4.03 & 20 & 0.68 \\
\hline
\end{tabular}

Como puede observarse, tomando como variable el género, se tuvo que las mujeres tienen una percepción de Riesgo percibido mayor $(M=2.88)$ que los hombres $(M=2.52)$, mientras que, al agrupar la muestra en usuarios $(M=3.66)$ y no usuarios de Facebook $(M=4.03)$, también se tuvieron diferencias de medias estadísticamente significativas en la escala de Utilidad percibida, ambos resultados significativos a $p<0.05$. No se encontraron diferencias estadísticamente significativas tomando en cuenta el tipo de dispositivo empleado para acceder a las redes sociales.

Continuando con los análisis no paramétricos, se realizaron correlaciones entre las escalas del instrumento empleando Rho de Spearman y se descartaron las correlaciones menores a 0.5 , obteniendo los resultados de la Tabla 6.

\section{Tabla 6}

Correlaciones estadisticamente significativas a $p=0.00$ entre las escalas del instrumento

\begin{tabular}{lll}
\hline Escalas & $\begin{array}{l}\text { Coeficiente Rho } \\
\text { Spearman }\end{array}$ \\
\hline Utilidad Percibida & Actitud & 0.59 \\
Utilidad Percibida & Intención de Uso & 0.65 \\
Intención de Uso & Actitud & 0.78 \\
\hline
\end{tabular}


José Manuel Meza Cano; Edith González Santiago

Como puede notarse, las correlaciones estadísticamente significativas se obtuvieron en las escalas de Utilidad Percibida, Actitud e Intención de uso, todas con un coeficiente mayor a 0.05, significativo a $p=0.00$. El resto de las escalas no presentaron correlaciones consideradas moderadas o fuertes.

\section{Conclusiones}

El objetivo del presente estudio fue describir la actitud, la utilidad percibida, la facilidad de uso, la confianza y el riesgo percibido de las redes sociales empleadas por estudiantes en línea de psicología, determinar en las variables sociodemográficas que pueden relacionarse con estas percepciones y determinar si existen correlaciones entre estas variables en la muestra.

Como era de esperarse, Facebook fue la red social más empleada por los estudiantes de la muestra, siendo utilizada varias veces al día por una amplia mayoría de ellos. Esto concuerda con los datos de Kemp (2020) a nivel global y también a nivel nacional. Algo que llama la atención es que los estudiantes también emplean con frecuencia WhatsApp que, si bien es un mensajero, se considera también como una red social, siendo empleadas varias veces al día por ellos.

En términos generales, la percepción de Utilidad, Facilidad de uso, Actitud hacia las redes e Intención de uso fueron favorables, mostrando medias cercanas a cuatro en una escala de uno a cinco, sin embargo, en las escalas de Riesgo percibido y Confianza, la percepción fue menos favorable, colocándose entre 2.6 y 2.7 en la misma escala. Por lo anterior, al parecer, los estudiantes de la muestra pueden encontrar las redes útiles, fáciles de emplear y pretenden seguir usándolas, pero son conscientes de los riesgos que se corren allí, lo que puede impactar también la confianza en ellas. Esto es importante de enmarcar en las características de la muestra, pues se trata de estudiantes en línea, con una media de edad de 35 años, por lo que no se trata de adolescentes, sino de adultos que inclusive pueden tener criterios de análisis sobre las desventajas de uso de las redes, como podría ser el ciberbullying (Varchetta et al., 2020) o el mal uso de la información de los usuarios (Guzmán, 2019). Esto se alinea con los hallazgos de García et al. (2018), quienes encontraron evidencia de que, con la edad, aumenta la percepción de la privacidad y su gestión, además de que la experiencia de uso aumenta la apreciación de riesgo en el uso de las redes; a su vez, Condor (2020) encontró que la percepción de alto riesgo en las redes sociales es liderada por Facebook con el $82 \%$ del total, seguida por Instagram con el $51 \%$ y WhatsApp con el 48\%, en una muestra de 202 estudiantes de educación superior.

Sin embargo, también la edad puede jugar en contra de la percepción de Facilidad de uso, dado que, conforme aumenta la edad, esta percepción se vio desfavorecida, lo cual puede tener relación con la aparente complejidad del uso de las redes sociales, las cuales incluyen funcionalidades y cambios en las interfaces continuamente, lo que es acorde con los hallazgos de Guzmán-Heras y Figueroa-Sacoto (2020), en donde encontraron diferencias entre jóvenes y adultos en la percepción de complejidad sobre redes como Facebook y WhatsApp.

Con respecto al género, se encontró que las mujeres tienen una mayor apreciación sobre Riesgo percibido al emplear las redes sociales. Los ítems de esta escala hacen referencia a la 
José Manuel Meza Cano; Edith González Santiago

percepción sobre el peligro, la seguridad y preocupaciones que puede generar el uso de la red social, por lo que es posible que las diferencias mostradas entre hombres y mujeres se deriven del tipo de interacción que muchas mujeres sufren al emplearlas, especialmente el ciberacoso y la violación de la intimidad. Por tanto, esta percepción de riesgo es menor en los hombres. Esto concuerda con los hallazgos de Domínguez et al. (2019), quienes encontraron una mayor frecuencia de varones ciberagresores, mientras que el impacto psicológico mayor es para las mujeres, generando, incluso, ideación suicida. En este sentido, Condor (2020) afirma que esto debe ser tomado en cuenta en las instituciones de educación superior, dado que, en ellas, cinco de cada diez mujeres y cuatro de cada diez hombres han sufrido violencia relacionada con el género en el espacio virtual, sintiendo inseguridad en estos medios.

Un hallazgo interesante es la diferencia entre aquellos usuarios de Facebook, quienes consideran que la Utilidad percibida es menor en relación con aquellos que no utilizan Facebook y emplean como red social principal WhatsApp, Instagram, u otras. Esto puede estar relacionado con la percepción de interacción que facilita la red, el acceso a la información y la utilidad en general, que son los ítems que componen a esta escala. ¿Cuál es la razón de que la mayoría de los usuarios de redes sociales empleen Facebook, pero tengan una baja percepción de utilidad? Cabe señalar que, de por sí, las medias de usuarios y no usuarios de Facebook se encuentran cercanas a cuatro, en una escala de uno a cinco, por lo que se consideran altas. Que los usuarios de otras redes sociales consideren que tienen una mayor percepción de utilidad puede estar relacionado con la finalidad de las propias redes, siendo Facebook una red social con utilidades diversas: tener contacto con familiares, amigos, mantenerse informado sobre noticias, eventos, etc. Mientras que las redes sociales como WhatsApp, para comunicarse con otros, e Instagram, para compartir fotografías, tienen un fin específico, esto se relaciona con los hallazgos de Cetinkaya y Sütçü (2018), quienes encontraron evidencia sobre las diferencias en el uso de Facebook y WhatsApp, encontrando en estudiantes preferencias sobre este último como medio de aprendizaje, mientras que Alhabash y Ma (2017) encontraron datos que aportan a este uso diferenciado, en donde los estudiantes mostraron mayores niveles de uso de Instagram con fines de entretenimiento, confort y autoexpresión, mientras que en Facebook mostraron una mayor disposición para compartir información, pero menor para la autoexpresión en estudiantes de educación superior.

Respecto a las correlaciones, los hallazgos muestran que la Utilidad percibida, la Actitud y la Intención de uso se encuentran relacionadas. Esto corrobora el modelo propuesto por Lorenzo et al. (2011), quienes ya habían dado cuenta de las relaciones que derivan en el uso de la red, en donde Intención de uso fue la variable que parecía relacionarse en mayor medida con las demás. Por lo que, si se requiere que los usuarios de redes sociales acepten una red, sería importante generar una buena Intención de uso, pues eso impactaría en la Actitud y la Utilidad percibida, aspectos que podrían tomarse en cuenta cuando se introducen nuevas redes sociales (Suárez et al., 2019).

Entre las limitaciones del estudio, se encuentra el tamaño de la muestra, que si bien es una muestra de 74 personas, no es una muestra representativa de la población de la carrera de 
psicología en línea, que se compone de alrededor de 3000 estudiantes activos; así como el tipo de muestreo, que en este caso fue por conveniencia, en vez de ser un muestreo aleatorizado. Aun con estas limitantes, el presente estudio abre una línea de investigación sobre la percepción de uso de las redes sociales en los estudiantes de psicología en línea, lo cual puede derivar en futuros estudios empleando, por ejemplo, redes sociales libres, de código abierto (Cabrera, 2017), actualmente denominadas redes sociales federadas (Liu et al., 2020), en donde se emplee una red para uso académico de profesores y estudiantes y así indagar en la percepción de uso de la misma a través del modelo TAM. En el contexto educativo, es importante, dado que es necesario brindar espacios virtuales seguros para la interacción entre docentes y estudiantes, quienes han buscado la integración de las redes sociales en el ámbito académico (Díaz, 2015; Flores et al., 2017), sin embargo, generalmente se trata de Facebook, Twitter o WhatsApp, cabe preguntarse si estos son espacios seguros y confiables para realizar actividades educativas y académicas, solamente por el hecho de ser las más empleadas.

Esto es relevante, dado que el uso y resguardo de los datos de los usuarios por parte de las redes sociales más comunes ha generado un gran debate, enfatizando sobre todo la posibilidad de cambiarse a otras redes más seguras. Inclusive, el Instituto Nacional de Transparencia, Acceso a la Información y Protección de Datos Personales (INAI, 2021) de México ha sugerido a los usuarios leer, analizar y tomar una decisión sobre el uso de WhatsApp derivado del cambio sus políticas de privacidad, esto a partir de la notificación de actualización de las condiciones del servicio del 4 de enero de 2021. Por lo tanto, es necesario, desde la academia y la universidad, no solo investigar en el uso de estos espacios virtuales, sino reflexionar críticamente sobre su alcance como medio de comunicación viable y confiable.

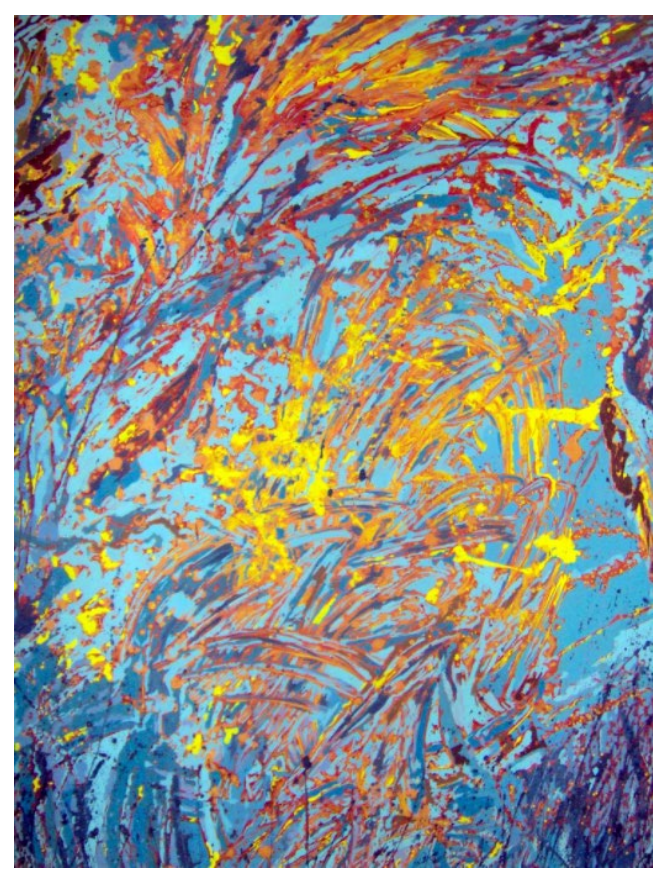

Desaforismo, acrílico sobre papel. Paula Boh 


\section{Bibliografía}

Alhabash, S. y Ma, M. (2017). A Tale of Four Platforms: Motivations and Uses of Facebook, Twitter, Instagram, and Snapchat Among College Students? Social Media + Society. https://doi.org/10.1177/2056305117691544 Asociación de Internet MX. (2019). 15 Estudio sobre los hábitos de los usuarios de internet en México 2018. Movilidad en el usuario de internet mexicano. Estadística digital. https://www.asociaciondeinternet.mx/estudios/habitos-de-internet Cabero, A. J., Barroso, O. J. y Gallego, P. O. (2018). La producción de objetos de aprendizaje en realidad aumentada por los estudiantes. Los estudiantes como prosumidores de información. Tecnología, Ciencia y Educación, (11), 15-46.

Cabrera, E. (2017). Redes sociales libres en la universidad pública. Revista Digital Universitaria, 18(1). http://www.revista.unam.mx/vol.18/num1/art06/

Cetinkaya, L. y Sütçü, S. S. (2018). The effects of Facebook and WhatsApp on success in English vocabulary instruction. Journal of Computer Assisted Learning, 34(5), 504-514. https://doi.org/10.1111/jcal.12255 Condor, B. A. (2020). Violencia de género en los espacios virtuales en los estudiantes de pregrado de la Facultad de Ciencias Administrativas de la Escuela Politécnica Nacional[Tesis de licenciatura]. Escuela Politécnica Nacional, Quito, Ecuador. https://bibdigital.epn.edu.ec/handle/15000/20925 Davis, F. D. (1989). Perceived usefulness, perceived ease of use, and user acceptance of information technology. MIS Quarterly, 13(3), 319-340. https://doi.org/10.2307/249008 Díaz, M. (2015). Importancia de las redes sociales en la educación. Medicina preventiva. http://medicinapreventivavn.blogspot.com/2015/08/importancia-de-las-redes-sociales-en-la.html Flores, L. G., Chancusig, C. J., Cadena, M. J., Guaypatín, P. O. y Montaluisa, P. R. (2017). La influencia de las redes sociales en los estudiantes universitarios. Boletín Redipe, 6(4), 56-65.

https://dialnet.unirioja.es/ejemplar/468338

Domínguez, R., Vargas, E., Castro, R., Medina, R. y Huerta, C. G. (2019). Ciberacoso como factor asociado al malestar psicológico e ideación suicida en adolescentes escolarizados mexicanos. Acta universitaria, 29. https://doi.org/10.15174/au.2019.2295

García, M., Del Olmo, B. y Del Hoyo, H. (2018) Jóvenes, privacidad y dependencia en las redes sociales. En Actas del II Congreso Internacional Move. net sobre Movimientos Sociales y TIC (pp. 128-138). Grupo Interdisciplinario de Estudios en Comunicación, Política y Cambio Social (COMPOLíTICAS). https://hdl.handle.net/11441/70629 Guzmán, S. C. (2019). Seguridad aplicada en la utilización de redes sociales. Repositorio Institucional Universidad Piloto de Colombia. http://repository.unipiloto.edu.co/handle/20.500.12277/6951 Guzmán-Heras, A. y Figueroa-Sacoto, T. (2020). Redes sociales en jóvenes y adultos mayores cuencanos. En A. Torres-Toukoumidis y A. De Santis (Coord.), Los morlacos y las redes sociales (pp. 90-107). Universidad Politécnica Salesiana.

https://dspace.ups.edu.ec/bitstream/123456789/19319/1/LOS\%20MORLACOS\%20Y\%20LAS\%20REDES\%20S OCIALES.pdf\#page $=90$

IFT (Instituto Federal de Telecomunicaciones). (2019). Uso de las TIC y actividades por internet en México. Impacto de las características sociodemográficas de la población (versión 2019). http://www.ift.org.mx/sites/default/files/contenidogeneral/estadisticas/usodeinternetenmexico.pdf 
INAI. (2021). INAI Ilama a usuarios de whatsapp a revisar a detalle nuevos términos de politica de privacidad y tratamiento de datos personales. https://home.inai.org.mx/wpcontent/documentos/SalaDePrensa/Comunicados/Comunicado\%20INAI-014-21.pdf

Kemp, S. (2020). Digital in 2020: New Decade, New Milestone. We Are Social. https://wearesocial.com/digital-2020

Liu, M., Gehl, R. W. y Zulli, D. (2020). Rethinking the 'Social'in 'Social Media': Insights into Topology, Abstraction, and Scale on the Mastodon Social Network. New Media and Society. http://dx.doi.org/10.17613/1mwy-vp43 Lorenzo, C., Alarcón, M. y Gómez, M. (2011). Adopción de redes sociales virtuales: ampliación del modelo de aceptación tecnológica integrando confianza y riesgo percibido. Cuadernos de Economía y Dirección de la Empresa, 14(3), 194-205. https://doi.org/10.1016/j.cede.2010.12.003 Luna, G. (2016). Las redes sociales virtuales como fuente de información y su influencia en el consumo simbólico de la experiencia turística [Tesis de doctorado]. Universidad de Sevilla. https://dialnet.unirioja.es/servlet/tesis?codigo $=61028$

Peña, E. y Sánchez, A. (2017). La integración de las redes sociales para el desarrollo de la competencia digital en la educación superior. Universitas Tarraconensis. Revista de Ciències de l'Educació, 7(1), 50-65.

http://dx.doi.org/10.17345/ute.2017.1.1782

Sánchez-Franco, M. J., Buitrago-Esquinas, E. M. y Hernández-Mogollón, J. M. (2012). Antecedentes sociales y psicológicos del compromiso comunitario. Un análisis del comportamiento del usuario de una red social de relaciones. Cuadernos de Economía y Dirección de la Empresa, 15(4), 205-220.

http://dx.doi.org/10.1016/j.cede.2012.04.007

Sánchez-Franco, M. J. y Roldán, J. L. (2015). The influence of familiarity, trust and norms of reciprocity on an experienced sense of community: an empirical analysis based on social online services. Behaviour \& Information Technology, 34(4), 392-412. https://doi.org/10.1080/0144929X.2014.959455

Suárez, R., Tijerina, A., Salas, G. y Escalona, M. (2019). Factores que influyen en la aceptación de la plataforma de enseñanza-aprendizaje Nexus en la Facultad de Filosofía y Letras de la UANL. Innovaciones de Negocios, 75(30). http://revistainnovaciones.uanl.mx/index.php/revin/article/view/253

UNESCO. (2014). Global citizenship education: Preparing learners for the challenges of the twenty-first century. http://unesdoc.unesco.org/images/0022/002277/227729E.pdf Varchetta, M., Fraschetti, A., Mari, E. y Giannini, A. (2020). Adicción a redes sociales, Miedo a perderse experiencias (FOMO) y Vulnerabilidad en línea en estudiantes universitarios. Revista Digital de Investigación en Docencia Universitaria, 14(1). https://dx.doi.org/10.19083/ridu.2020.1187

Vidal, M. E. (2018). Determinantes de la aceptación del mobile learning como elemento de formación del capital humano en las organizaciones [Tesis doctoral]. Universidad Complutense de Madrid. https://eprints.ucm.es/46166/

\footnotetext{
'Estudio realizado gracias al proyecto PAPIIT "Creencias Epistemológicas Específicas a Internet y su relación con la discriminación de noticias falsas en redes sociales" <|A302121〉.
} 